\title{
Evaluation of spectrum opportunities in the GSM band
}

\author{
Andrea Carniani ${ }^{\# 1}$, Lorenza Giupponi ${ }^{2}$, Roberto Verdone ${ }^{\# 3}$ \\ \# DEIS - University of Bologna, viale Risorgimento, 2 - 40136, Bologna, ITALY \\ *Centre Tecnòlogic de Telecomunicacions de Catalunya (CTTC), Av. Canal Olímpic s/n 08860, Castelldefels (Barcelona), SPAIN \\ ${ }^{1}$ andrea.carniani@unibo.it, ${ }^{2}$ Iorenza.giupponi@cttc.es, ${ }^{3}$ roberto.verdone@unibo.it
}

\begin{abstract}
In this paper we explore the possibility of exploiting underutilized channels in the GSM bands. The secondary (unlicensed) users of the spectrum are the nodes of an overlay cognitive ad-hoc network, which opportunistically transmits in data channels left unused by the primary (licensed) system. The fundamental constraints for the secondary system are: 1) it uses the time slots/frequency channels (i.e. data channels) unused by the primary GSM system, 2) its operation does not degrade the performances of the primary system, 3) there is no exchange of signalling information between the primary and the secondary system to facilitate the secondary usage of spectrum, that is, the primary users are not cognitive aware. The focus is on the feasibility of the proposed approach, so that we consider a system level simulator based on a realistic GSM network deployed in the city of Bologna. In this context we evaluate the impact that the operation of the secondary users has on the performances of the primary system. In addition, we study the performances that can be obtained by the secondary system under the condition of marginal interference to the primary system. We will show that an appropriate choice of the frequency channel, makes the secondary usage of spectrum a valid approach to increase the operator's spectrum efficiency.
\end{abstract}

\section{INTRODUCTION}

Today's wireless networks are characterized by a fixed spectrum assignment policy; however, a large portion of the assigned spectrum is used sporadically and geographical variations in the utilization of assigned spectrum ranges from $10 \%$ to $85 \%$ with a high variance in time. With the objective of enhancing the utilization of the limited spectrum resources, a new paradigm in wireless communications has been defined, referred to as Cognitive Radio (CR). The basic idea is that a secondary user (a cognitive unlicensed user) is able to properly sense the spectrum conditions and, to increase efficiency in spectrum utilization, it seeks to underlay, overlay or interweave its signals with those of the primary (licensed) users, without impacting their transmission [1]. In this paper we explore the possibility of exploiting the underutilized channels in the GSM band for secondary adhoc communications between secondary (unlicensed) users without causing harmful interference to the primary system. In this context, we focus on drawbacks and advantages of the interweave and underlay paradigms, as they have been defined in [1]. The interweave paradigm for cognitive radio is based on the idea of opportunistic communications. The idea is to exploit the temporary space-time frequency voids, referred to as spectrum holes, which are not in constant use in both licensed and unlicensed bands, for a secondary use of spectrum. The underlay paradigm, on the other hand, encompasses techniques that allow concurrent primary and secondary transmissions, as long as the interference generated by the secondary users is below some acceptable threshold.

In order to select the most appropriate data channel where to realize the secondary transmission, the secondary users operation follows four steps: (1) spectrum sensing is first activated to determine and characterize the available radio resources in the different frequency channels; (2) spectrum decision exploits the information collected during spectrum sensing to characterize the available spectrum holes, in order to select the most appropriate data channel to achieve an efficient secondary usage of spectrum; (3) spectrum sharing promotes the coordinate scheduling among secondary users coexisting in the same cell; (4) spectrum handoff is defined as the process that occurs when a secondary user changes its data channel: it may occur when the current data channel conditions become worse, or when a primary user appears in the data channel that the secondary is using for communications. Thus, based on the results collected during spectrum sensing, the spectrum has to be properly managed to: 1) guarantee effectiveness in the secondary usage of spectrum, 2) not cause harmful interference on the primary receivers through secondary communications, 3) meet, as much as possible, the secondary communications requirements.

In this paper, we first compare the interweave and underlay paradigms, to learn drawbacks and advantages of each choice. Then, focusing on the underlay paradigm, we address the spectrum decision problem and we study the impact of secondary communications on primary and secondary systems. Preliminary simulation results, obtained by means of a realistic C simulator of the GSM network of an Italian operator in the Bologna area, show that by means of appropriate spectrum decisions, the spectrum can be reused taking advantage of the traditional GSM frequency planning.

The outline of this paper is organized as follows. Section II compares two paradigms for cognitive radio and selects the most appropriate one for secondary use in GSM bands. Section III describes the proposed spectrum decision scheme. Section IV presents the considered simulation tool. Section V describes the simulation scenario and the preliminary simulation results. 
Finally Section VI summarizes the main conclusions and future works.

\section{INTERWEAVE VERSUS UNDERLAY}

In this section, taking into account the characteristics of the GSM band, we compare the interweave and underlay paradigms for secondary use in this band.

The interweave paradigm for cognitive radio is based on the idea of opportunistic communications, and was the original motivation for cognitive radios. The idea came about after observing the existence of temporary space-time frequency voids, referred to as spectrum holes, which are not in constant use in both licensed and unlicensed bands. These gaps change with time and can be exploited by SUs for their communications. This approach is used in [2][3] where the authors evaluate different spectrum decision schemes to access GSM Downlink (DL) frequency carriers. In this case the victims of the interfering secondary communication are the GSM mobile terminals. This approach can be considered viable in an urban scenario since secondary transmitters and primary receivers would be at the same height level, so that Non Line of Sight (NLOS) in urban areas can be exploited. However, since NLOS cannot be assumed for all the situations, the secondary transmission has to be perfectly synchronized with the primary transmission in order not to cause harmful interference to the primary receivers. This perfect synchronization is not possible to implement due to delay in the spectrum sensing operation. In particular, in [2] the authors show that depending on the relative positions of the primary and secondary users, and depending on their positions with respect to the centralized base station, an overlap period between primary and secondary transmissions active in contiguous slots, may occur with a duration of $\tau=\tau_{B S-S U}-\tau_{B S-P U}+\tau_{S U-P U}$, where $\tau_{B S-S U}, \tau_{B S-P U}$ and $\tau_{S U-P U}$ represent the propagation delays between the base station and the secondary user, the base station and the primary user, the primary user and the secondary user, respectively. As a result, a potential interference always exists during a fraction of the time slot. In this case, to reliably estimate the interference that the secondary transmission would cause to the primary receivers via reciprocity, a sensing operation is required during at least $480 \mathrm{~ms}$, since a measure over the time slot is not considered reliable due to the fading effect. In addition, the handshake operation of the secondary users, realized by means of exchange of RTS (Request To Send)/CTS (Clear To Send) messages, needs to be transmitted in a common control channel (CCC), but the existence of such a control channel has often been rejected in the cognitive radio research community, since it requires a static assignment of licensed spectrum before deployment, which is basically against the same philosophy of cognitive radio. Finally, it is worth mentioning that differently from other bands considered in literature for secondary usage (i.e. Television bands), GSM is not an underutilized frequency band, so that the percentage of unused slots is not so high to motivate this kind of opportunistic approaches. On the other hand, it would make much more sense to take advantage of the planning of GSM frequency carriers, which was realized by operators accounting for spatial reuse.

The underlay paradigm mandates that concurrent noncognitive and cognitive transmissions may occur only if the interference generated by the cognitive devices at the noncognitive receivers is below some acceptable threshold: this approach is used in [4] where the authors consider an overlapping secondary communication in the Uplink (UL) frequencies, so that the victim of this approach would be the GSM base station. The advantage of this approach is that the position of the secondary receiver is known (in fact by measuring the $\mathrm{BCCH}$, transmitted at maximum constant power, it is possible to estimate the position of the base station); however, a secondary communication would easily interfere with the base station since, differently from the previous case, the secondary user is always in line of sight (LOS). Additionally, it should be mentioned that none of the approaches available in literature considers the operation of frequency hopping (always implemented in real GSM systems) in the design of the opportunistic approach.

As a result of these considerations, in this paper we propose that the opportunistic access to GSM unused frequency channels can be improved by using the UL frequency carriers and considering the underlay approach. The underlay paradigm is selected as the most appropriate paradigm for secondary use of GSM bands, so as to realize sensing over $480 \mathrm{~ms}$ and to avoid the need for being perfectly synchronized with the GSM frame structure. To avoid interference with multiple GSM terminals or with the GSM base stations, we propose to take advantage of the GSM frequency planning, by opportunistically accessing to the GSM frequency carriers not allocated in the cell where the secondary users are operating.

\section{SPECTRUM DECISION}

In this paper we evaluate secondary spectrum opportunities in the GSM band, by considering realistic data. In particular, we focus on giving guidance for spectrum decision in the defined scenario. We propose that every time a secondary user has to select a frequency channel for transmission, it follows a procedure based on four steps, which are described in the following.

1) Context awareness of secondary user's cell: The secondary user listens to the $\mathrm{BCCH}$ (Broadcast Control Channel) of the cell where it is located, $B C C H_{0}$, to get the following information:

- Frequency allocated in that cell;

- BCCH of the surrounding cells;

- SCH (Synchronization Control Channel);

- Frequency hopping sequences;

We consider $480 \mathrm{~ms}$ to access to this information, in order to get reliable measurements over the $\mathrm{SACCH}$ period. Since the $\mathrm{BCCH}$ period is $235 \mathrm{~ms}$, it will be possible to listen during a complete $\mathrm{BCCH}$ channel. 
2) Context awareness of surrounding cells: the secondary user listens to $\mathrm{BCCH}$ of the surrounding cells to get the following information:

- Frequency carriers allocated in surrounding cells;

- SCH to synchronize to frame structure;

- Frequency hopping sequences.

To access to this information $480 \mathrm{~ms}$ are required to listen to $8 \mathrm{BCCH}$, in the optimal case of perfect desynchronization among different $\mathrm{BCCH}$. This hypothesys is not realistic, so the secondary user will probably need at least $480 \mathrm{~ms} \times 2$ to listen to $8 \mathrm{BCCH}$.

Notice that these two preliminary steps can be realized offline, so that the secondary user can dispose of this information in the moment it switches on, since this is static information related to the frequency planning. The other steps of the proposed algorithm are defined as follows:

3) Spectrum sensing: If some of the UL frequency carriers are not used in the surrounding cells, one of them is selected for secondary transmission. The rationale behind this choice is that the base station receiving in that frequency is not close to the secondary transmitter, so that the secondary transmission would not cause harmful interference to it. If in turn all frequency carriers are used in the cells surrounding the secondary user, the three cells characterized by the three $\mathrm{BCCH}$ received with lowest power are identified and indicated in the following by the corresponding $\mathrm{BCCH}$ channels, as $\mathrm{BCCH}_{i}, i=$ $1,2,3$. Sprectrum sensing is then realized with respect to the frequencies allocated in the three identified cells. The sensing is realized in the UL carriers, in order to gain information about the resource availability and aggregated interference generated by both secondary and primary users.

4) Spectrum analysis and spectrum decision: If the power received by $B C C H_{0}$ is much higher than that received by $B C C H_{i}, i=1,2,3$, it means that the secondary user is close to its base station, so that the frequency channel for secondary transmission can be selected among those allocated for the UL in the cell characterized by the worse $\mathrm{BCCH}$. The selection of the target frequency carrier is carried out by taking into account information about resource availability and perceived aggregated interference. On the other hand, if the power received from one of $B C C H_{i}, i=1,2,3$ is lower than the others, then the cell associated with this $\mathrm{BCCH}$ can be selected as target cell, and the target frequency channel can be selected among those allocated for UL transmission in this cell. The information to take into account is the resource availability and perceived aggregated interference in each frequency channel. Finally, if in turn the secondary user finds itself in a situation where the received power levels from the $\mathrm{BCCH}$ of the surrounding cells are almost comparable, we assume to be in an urban environment. As a result, among all available frequency channels in the three surrounding cells we select a frequency carrier in the DL to take advantage of the NLOS.

\section{Simulation TOOL}

In this section we briefly introduce the simulation tool used to implement and evaluate the performance of the proposed spectrum decision algorithm. This tool has been developed at University of Bologna and it is a dynamic $\mathrm{C}$ simulator for cellular networks. In particular this simulator evaluates the performance of the real GSM network of an Italian operator in the Bologna area, taking into account the mobility of GSM's users, the propagation aspects, the amount of useful power, the interference and the radio resource management algorithms (power control, handover, channel allocation, etc).

This simulator is composed of two main parts: the traffic simulator and the network simulator. The traffic simulator takes as inputs the number of users and the number of base stations, and it provides as outputs, the beginning and the end of each call, and the mobility patterns of the users during the simulation. In particular, the users are classified according to their speed (e.g. random-walk pedestrian, random-walk vehicular, street-walk vehicular, mixed) and class type.

The network simulator, which is the core of the entire tool, aims to obtain the KPIs (Key Performance Indicators) to evaluate the performances of the entire GSM network. It takes as inputs:

- User's movement file and call generation file: They are the traffic simulator's output, and represent the amount of traffic within the GSM network and users' mobility patterns within the scenario;

- base station file: it contains information about the position of the base stations, pointing angle of antennas, the type of antenna and the list of surrounding and interfering base stations;

- frequency hopping file: it contains information about carrier distribution among the frequency hopping groups for each base station;

- frequency allocation file: it contains information about frequency carriers allocation among the base stations.

The network simulator implements frequency planning with bandwidth segregation to obtain better performances of the entire system. In addition, the simulator implements all radio resource management strategies: power control, frequency hopping, admission control, link adaptation, load control and handover. It uses the locating handover which guarantees call quality checking at the cell border to minimize the overall interference into the network. Finally, the network simulator provides the spectrum occupancy of the GSM band in the considered scenario. In particular, the simulator provides the channels (frequency carrier/time slot) that are used by the primary users (mobile terminal in UL, base station in DL). We consider that this information, together with information about the aggregated interference from the primary users, is available to the secondary users after executing the spectrum sensing phase.

The block diagram of the network simulator illustrated is shown in Fig. 1 


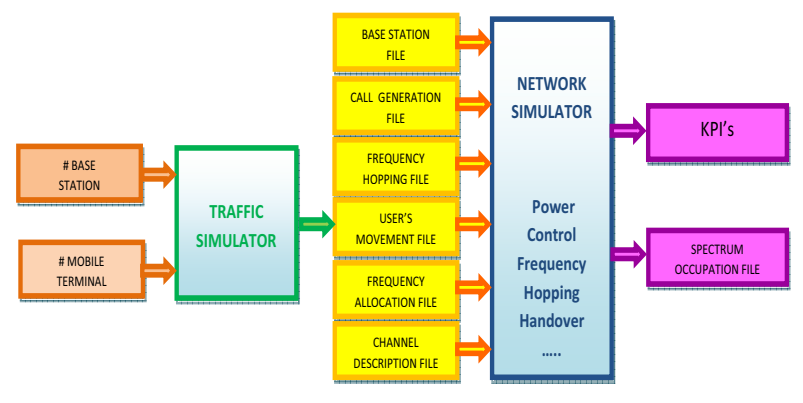

Fig. 1. Block Diagram of simulation tool.

\section{SimUlation SCENARIO AND PRELIMINARY RESULTS}

As already discussed in section II, we evaluate the possibility of exploiting underutilized channels in the GSM band, taking advantage from traditional GSM frequency planning, in a realistic setting provided by the GSM network of an Italian operator in the Bologna area, whose best servers'map is shown in Fig. 2.

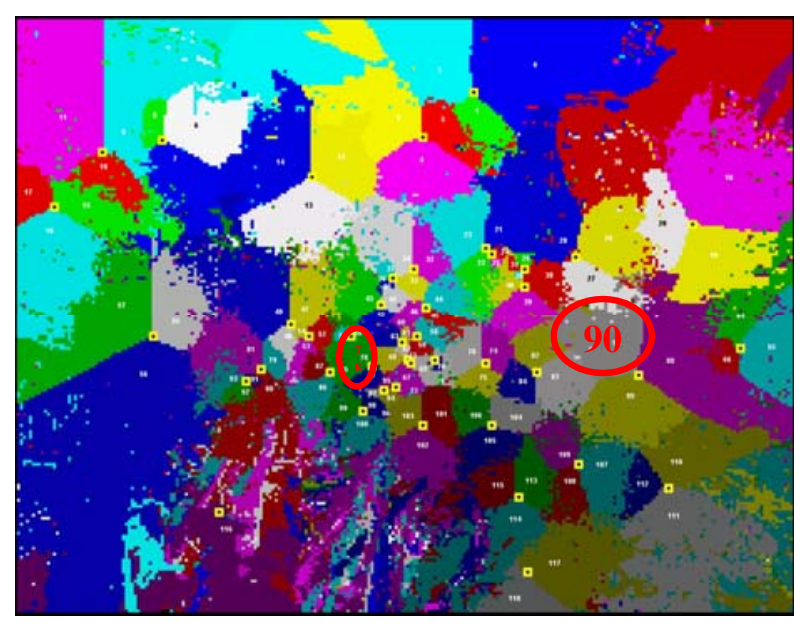

Fig. 2. Best servers'map in the area of Bologna.

We locate a pair of secondary users in the scenario depicted in Fig. 2, and we evaluate the SINR (Signal to Interference plus Noise Ratio) at the primary and secondary users' receiver. We consider both the situations where secondary users are operating in urban and sub-urban areas, in order to learn the impact that the surrounding environment has on the primary and secondary performances. In particular, we will focus on cell 90, for the sub-urban case, and on cell 78 for the urban case (see Fig. 2). For all simulation results contained in this paper, we assume that the duration of secondary transmission is 2 minutes and that the SUs have to transmit data blocks of $16 \mathrm{~KB}$ (so they transmit continuously in each superframe interval of $480 \mathrm{~ms}$ ). In addition we consider a constant secondary transmission power $P_{S U}=10 \mathrm{dBm}$.

\section{A. Evaluation of primary system's performaces}

In the sub-urban case, after the two context awareness phases described in section III, we consider that the secondary transmitter randomly selects one of the frequency channels not allocated in the surrounding cells, since some of them are available in this part of the scenario. We compare the SINR measured at each base station (primary receiver) that is using the frequency carrier selected by the secondary users (i.e. cells 7, 72 and 76), when secondary communications are active and when they are not. Simulation results show that secondary transmissions do not modify the SINR perceived by the primary system. The reason is that the base stations receiving in the selected frequency channel are not close to the secondary transmitter, so that the secondary transmission does not cause harmful interference to it. This result is shown in Fig. 3, where the Cumulative Distribution Function (CDF) of the difference between the SINR measured without secondary activity and the SINR measured in presence of secondary activity (i.e. deltaSINR) is plotted for all the cells that are experiencing interference due to secondary operation.

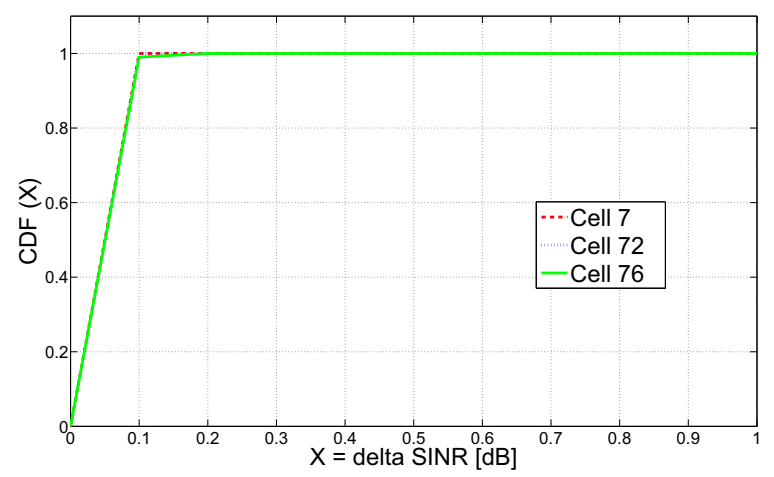

Fig. 3. CDF of deltaSINR for the SUB-URBAN case - secondary user's frequency carrier is not allocated in the surrounding cells.

We replicate the same experiment for the urban case. As discussed before, during the context awareness phases, the secondary users discover that some frequency carriers are not allocated in the surrounding cells so that they choose randomly one of these frequency carriers allocated for UL primary transmission. We evaluate the CDF of deltaSINR with the measures realized in the not surrounding cells 69 and 80 , which are using the frequency carrier selected by the secondary user. The corresponding simulation results are shown in Fig. 4.

It can be observed that the impact of the secondary transmission is higher than in the sub-urban case, because in the urban scenario the cells are smaller, so that the not surrounding cells where the selected frequency channel may be used, could be quite close to the position of the secondary transmitter. However, we observe that the SINR performances of the primary base stations are still high (i.e. the average SINR with secondary user's activity is $37.04 \mathrm{~dB}$ for cell 69 and $32.14 \mathrm{~dB}$ for cell 80 , while with no secondary user's activity is 40.47 


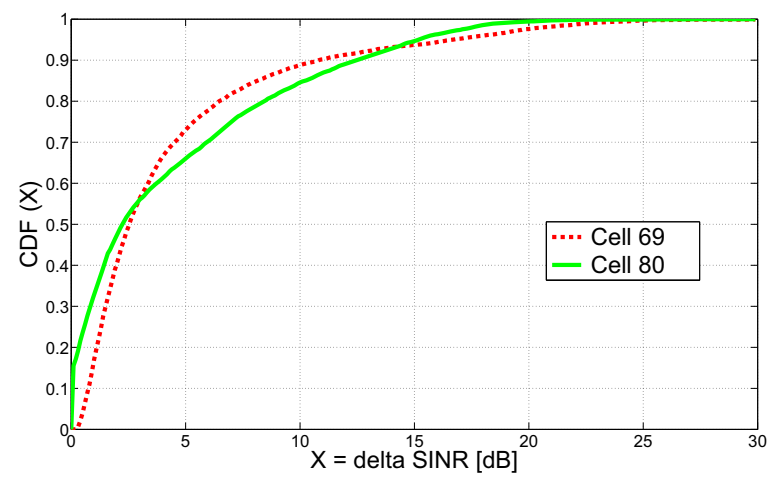

Fig. 4. CDF of deltaSINR for the URBAN case - secondary user's frequency carrier not allocated in the surrounding cells.

$\mathrm{dB}$ for cell 69 and $36.57 \mathrm{~dB}$ for cell 80 ).

After verifying that the secondary activity does not degrade the performances of the primary system frequency channels not allocated in the surrounding environment are selected, we focus on evaluating system performances, when the secondary user selects a frequency channel used in the surrounding cells. Again, we focus on both sub-urban and urban cases, i.e. on cells 90 and 78, respectively. In this case, we force the secondary users to choose randomly one of the frequency carriers allocated in one of the secondary users' surrounding cells. Fig. 5 and 6 depict the CDF of deltaSINR in the suburban and urban cases, respectively. It can be observed that, as it was expected, the increment of interference generated by the secondary transmission is very high in the urban case, especially for those cells close to the secondary user's cell. In particular, the average SINR measured by the primary receiver, in presence of secondary user transmissions, is $23.67 \mathrm{~dB}$, in cell $63,40.73$ in cell 79 , and $21.89 \mathrm{~dB}$ in cell 102 , while it is $35.9 \mathrm{~dB}$ in cell $63,45.52$ in cell 79 , and $23.92 \mathrm{~dB}$ in cell 102 , without secondary user's activity. On the other hand, in the sub-urban case the increment of harmful interference is reduced with respect to the urban case, since on average the surrounding cells are characterized by greater coverage areas, so that the primary receivers are in general further from the secondary transmitters. In fact in this case, we observe that the average SINR measured by the primary receiver is 51.19 $\mathrm{dB}$ and $59.73 \mathrm{~dB}$ in cell $26 ; 60.68 \mathrm{~dB}$ and $61.05 \mathrm{~dB}$ in cell $61 ; 37.88 \mathrm{~dB}$ and $37.91 \mathrm{~dB}$ in cell $84 ; 43.23 \mathrm{~dB}$ and 43.23 $\mathrm{dB}$ in cell 103, in presence of secondary users and without secondary activity, respectively.

\section{B. Evaluation of secondary systems's performances}

To evaluate the performances of the secondary system, we consider that the interfering users are the mobile terminals transmitting in the same frequency carriers chosen by the secondary users. Due to the frequency hopping, each frequency channel is not continually used by a primary user, during the $480 \mathrm{~ms}$ superframe interval, so that to evaluate the interference measured by the secondary receiver, we need to make different assumptions. We indicate with $K$ the number of primary users

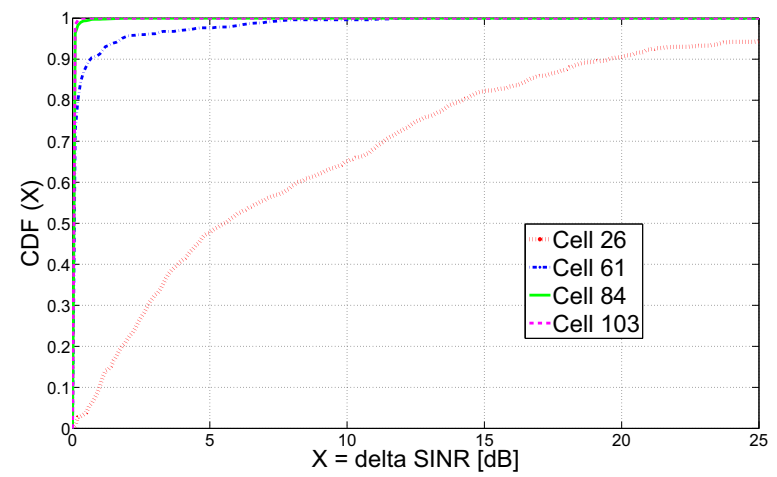

Fig. 5. CDF of deltaSINR for the SUB-URBAN case - secondary user's frequency carrier allocated in one of surrounding cell.

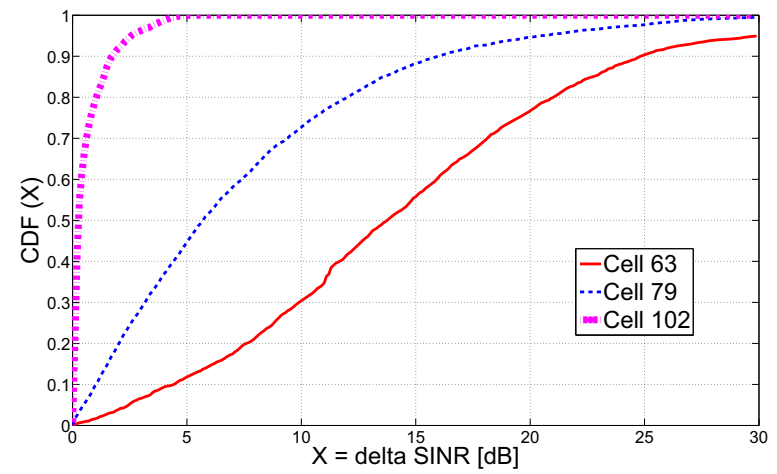

Fig. 6. CDF of deltaSINR for the URBAN case - secondary user's frequency carrier allocated in one of surrounding cell.

assigned to a given frequency carrier $\mathrm{F}$ in cell $i$, and with $n_{j}$ the number of time slots in $\mathrm{F}$, allocated to primary user $j$, with $j=1, \ldots, K$. We define $n$ as the primary activity on $\mathrm{F}$, expressed in time slots:

$$
n=\sum_{j=1}^{K} n_{j}
$$

As a result, the activity factor of the frequency carrier $\mathrm{F}$, allocated in cell $i$ during the superframe interval is:

$$
\alpha_{i}=\frac{n}{n_{\text {frame }} \cdot n_{\text {slot }}}
$$

where $n_{\text {frame }}=104$ is the number of frames in $480 \mathrm{~ms}$ and $n_{\text {slot }}=8$ is the number of slots per frame. To evaluate the average interference on the secondary user receiver, generated by the primary transmissions from interference cell $i$, we use the following expression:

$$
\widehat{I_{S U}^{B S}}=\alpha_{i} \cdot I_{S U}^{B S_{i}}
$$

where $I_{S U}^{B S_{i}}$ is the expected value of the total interference generated by primary transmitter (mobile station) $j$ and received by the secondary receiver, $I_{M S_{j}}$. 


$$
\begin{array}{r}
\widehat{I S S}_{S U}^{B S}=E\left[I_{M S_{j}}\right]=\frac{1}{n} \cdot\left\{\sum_{j=1}^{K} I_{M S_{j}}\right\}= \\
=\frac{1}{n} \cdot\left\{\sum_{j=1}^{K}\left[\sum_{l=1}^{n_{j}} I^{M S_{j l}}\right]\right\}
\end{array}
$$

Assuming that the number of interfering cells is $N$, the average interference measured by the secondary receiver is:

$$
I_{S U}=\sum_{i=1}^{N} \widehat{I_{S U}^{B S}}
$$

With respect to the channel model from the primary transmitter $j$ to the secondary receiver, and between secondary transmitter and secondary receiver, we consider a pathloss model depending on the distance:

$$
L(d B)=k 0+k 1 \ln d+s,
$$

where $k 0=31.61 d B$ and $k 1=12.39$ depend on the propagation environment, $d$ is the distance between primary users $j$ active in interfering cell $i$, and secondary user receiver, or between the nodes of the secondary users' pair, and $s$ is the log-normal shadowing contribution defined based on an exponential model as in [6]. For the noise power, we use a constant value $P_{N}=-113 \mathrm{dBm}$.

To evaluate the performance in terms of SINR we consider different scenarios where we vary the position of the secondary transmitter, and the secondary receiver, on circumferences whose center is the position of the secondary transmitter and the radius is the distance $d_{u}$ among the two nodes. In particular, we evaluate the performance with 5 different positions of the secondary transmitter and for each of them, 5 different positions of the secondary receiver. We consider the same cases analyzed for the primary system, so that in Fig. 7 we show the SINR measured at the secondary receiver in the following cases:

A Secondary users located in a sub-urban cell (cell 90) and secondary transmitter chooses one of the frequency carrier not allocated in surrounding cell;

B Secondary users located in a sub-urban cell (cell 90) and secondary transmitter chooses one of the frequency carrier allocated in surrounding cell;

C Secondary users located in a urban cell (cell 78) and secondary transmitter chooses one of the frequency carrier not allocated in surrounding cell;

D Secondary users located in a urban cell (cell 78) and secondary transmitter chooses one of the frequency carrier allocated in surrounding cell.

On the one hand, it can be observed that the average SINR of the secondary transmission is always characterized by high values, for the sub-urban cases, since the primary transmitters are on average, far from the secondary users. Besides, it should be noticed that the SINR measured in case $A$ is similar to that measured in case $B$. The reason is that the distance between secondary receiver and primary transmitter is still high in case $B$, and the activity factor in the interfering cell is higher in case $B$ than in case $A$. In fact, the average activity factors are

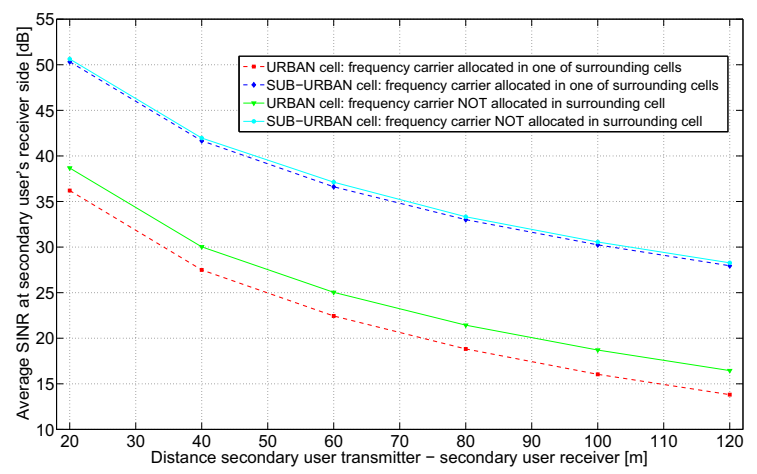

Fig. 7. Average SINR.

- case A: 0.1 for cell 7, 0.03 for cell 72, 0.09 for cell 76;

- case B: 0.06 for cell 26, 0.001 for cell $61,0.008$ for cell 84, 0.002 for cell 103 .

On the other hand, for the urban cases the SINR is lower than the sub-urban cases, and the difference between cases $C$ and $D$ is now much more significant. The reason is that in case $D$, the primary transmitters are close to the secondary receivers, and the activity factors are comparable in interfering cells. In these cases the average activity factors are:

- case C: 0.009 for cell 69, 0.14 for cell 80;

- case D: 0.08 for cell 26, 0.45 for cell $61,0.04$ for cell 84 .

Finally, we evaluate the maximum distance which guarantees good communication performances between the secondary transmitter and the secondary receiver, for each of the cases presented above. In particular, we set to $10 \mathrm{~dB}$ the minimum value of acceptable SINR for the secondary system, and we accept to have $S I N R<10 d B$ for 10 per cent of cases. Fig. 8 shows the probability that the SINR at the secondary receiver is below the threshold (i.e. outage probability), as a function of the distance between nodes. The lowest maximum distance is obtained for case $D$, where the secondary users in the urban scenario choose one of the frequency allocated in the surrounding cells (in particular in case $D$ we have a maximum secondary user's transmission range between $80 \mathrm{~m}$ and $90 \mathrm{~m}$ ). Hence we can say that, especially in urban cells, it is important to choose in an accurate way the frequency carrier for secondary use to obtain good performance for the secondary transmission and to avoid significant interference to the primary system.

\section{CONCLUSION AND FUTURE WORKS}

In this work we have evaluated spectrum opportunities for secondary use in a realistic GSM network deployed by an Italian operator in the Bologna area. We have carried out an extensive simulation work in order validate the hypothesis that the GSM frequency bands can be exploited for secondary use of spectrum, by taking advantage of the traditional GSM frequency planning. The preliminary results show that there is the possibility for a secondary usage of GSM bands, 


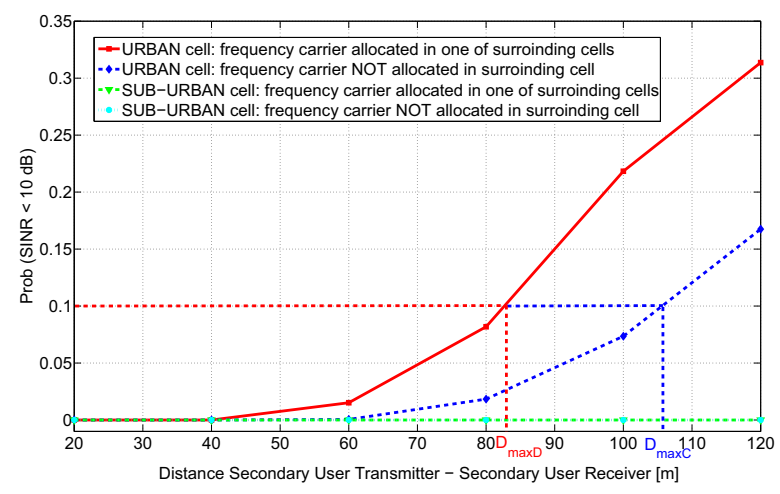

Fig. 8. Secondary users' outage probability.

without causing harmful interference to the primary system and providing good performances for the secondary system. However, appropriate algorithms for spectrum decision have to be designed, taking into account multiple aspects such as the characteristics of the cell where the secondary users are located (i.e. urban or sub-urban), the activity factor of the primary users in the available frequency channels, the distance between secondary transmitter and receiver. The results shown are referred to a very simple scenario, with only two pairs of secondary users. In future works we will design a comprehensive spectrum decision algorithm capable of making decisions in this scenario in such a way to provide good performances to secondary receivers without creating harmful interference to the primary system.

\section{ACKNOWLEDGMENT}

This work was supported by the European Commission in the framework of the FP7 Network of Excellence in Wireless COMmunication NEWCOM++ (contract n.216715).

\section{REFERENCES}

[1] A. Goldsmith, S. A. Jafar, I. Maric, S. Srinivasa, "Breaking Spectrum Gridlock with Cognitive Radios: an Information Theoretic Perspective", Proceedings of the IEEE, invited, to appear 2008.

[2] S. Sankaranarayanan, P. Papadimitratos, A. Mishra, S. Hershey, A bandwidth sharing approach to improve licensed spectrum utilization, in Proc. IEEE Dyspan 2005, PP. 279-288, Nov. 2005.

[3] L.Marin, L.Giupponi "Performance evaluation of spectrum decision schemes for a Cognitive Ad-Hoc Network", in IEEE PIMRC 2008.

[4] B. Kaufman, B. Aazhang, "Cellular Networks with an Overlaid Device to Device Network", IEEE Asilomar Conference on Signals, System and Computers, 2008.

[5] I.F.Akyildiz, Won-Yeol Lee, M. C. Vuran, S. Mohanty "A survey on spectrum management in cognitive radio networks", IEEE Communication Magazine, vol.7, pp. 40-48, April 2008.

[6] M. Gudmundson, "Correlation model for shadow fading in mobile radio systems", IEEE Electronics Letters, vol. 27, pp. 21452146, Nov. 1991. 